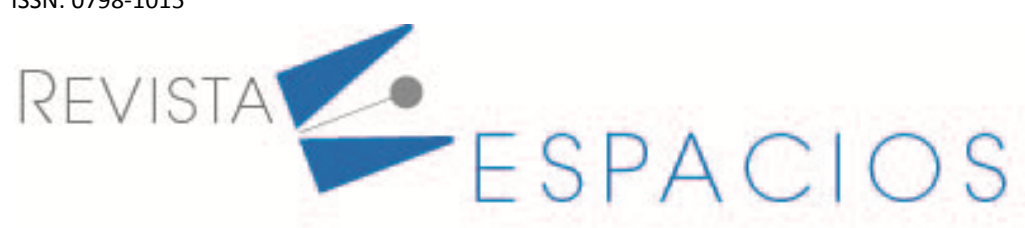

\title{
Factores que influyen en la calidad de la educación virtual
}

\section{Factors influencing the quality of virtual education}

MARTELO, Raúl J. ${ }^{1}$

FRANCO, David A. ${ }^{2}$

OYOLA, Paulo $\mathrm{S}^{3}$

\begin{abstract}
Resumen
Se identificaron y clasificaron factores que influyen en la calidad de la educación. Metodológicamente el estudio fue de tipo mixto con diseño no experimental, transversal, correlacional, y descriptivo. Teniendo en cuenta lo anterior, se aplicaron las técnicas revisión sistemática y MIMAC para obtener información sobre los factores que intervienen en la calidad de la educación, y realizar un análisis estructural. Respecto a los resultados, se obtuvieron factores como la autogestión de los contenidos de aprendizaje, y Metodología de trabajo.
\end{abstract}

Palabras clave: virtualidad, educación, TIC, aprendizaje

\begin{abstract}
Factors influencing the quality of education were identified and classified. Methodologically, the study was mixed with a non-experimental, cross-sectional, correlational, and descriptive design. Taking into account the above, the systematic review and MIMAC techniques were applied to obtain information about the factors that intervene in the quality of education, and to carry out a structural analysis. Regarding the results, factors such as self-management of learning contents and work methodology were obtained.

key words: virtuality, education, ICT, learning
\end{abstract}

\section{Introducción}

El desarrollo tecnológico ha transformado a las naciones en sus contextos sociales, económicos, culturales y educativos (Medina, 2014). La introducción de las TIC en el área educativa, por ejemplo, contribuye a la ruptura de los modelos tradicionales de enseñanza y origina nuevas formas como la educación virtual, la cual no sólo supone el cambio de la tiza por aplicativos de equipos informáticos, sino la oferta de mecanismos útiles para que los estudiantes adquieran conocimientos de manera autónoma (Niño, 2018). En este sentido, las TIC son un vehículo que permite el acceso a la educación a personas que de otro modo no podrían acceder (Román, 2018). Esto es debido a que estas herramientas ofrecen facilidad en la interacción entre docentes (tutores) y

\footnotetext{
${ }^{1}$ PhD (e) - Magister en Informática. Líder del grupo de investigación INGESINFO. Profesor de planta del Programa Ingeniería de Sistemas de la Universidad de Cartagena. e-mail: rmartelog1@unicartagena.edu.co

2 Magister en Ciencias Computacionales. Líder del grupo de investigación GIMATICA. Profesor de planta del Programa Ingeniería de Sistemas de la Universidad de Cartagena. e-mail: dfrancob@unicartagena.edu.co

${ }^{3}$ Docente. Facultad de Ciencias Económicas. Universidad de Cartagena. e-mail:poyolaq@unicartagena.edu.co
} 
estudiantes, reduciendo barreras de horarios y distancias, además de suministrar una enorme cantidad de información, con altos grados de eficiencia y a un bajo costo (Sierra, 2011).

A pesar de las bondades, la educación virtual no ha tenido importancia que merece, sin embargo, también es valorada de forma significativa por otros colectivos (Barberá, Badia, \& Mominó, 2001), y aún más si se tiene en cuenta la época de pandemia de COVID-19 en la cual fue el único modo para dar continuidad con la educación, debido a los continuos confinamientos, cuarentenas y restricciones emitidas por los gobiernos. Considerando la situación descrita, es necesario aclarar que la educación virtual requiere de un alto nivel de calidad que contribuya al desarrollo de las competencias de los educandos (Ardila-Rodríguez, 2011). Algunos autores afirman que la calidad de la educación virtual (CEV) se relaciona con la realización de la docencia en diversos espacios de aprendizaje (Marúm-Espinosa, 2011), mientras que en la Norma UNE 66181 se expone que se asocia con la satisfacción de los usuarios y su formación integral. Por su parte, para Sánchez (2012), la educación virtual es de calidad si ofrece lo que realmente dice ofrecer.

Las anteriores definiciones describen la CEV, sin embargo, el estudio de este campo supone grandes desafíos a investigadores, docentes y gestores de dicha modalidad de estudio, debido a las dificultades para comprender los factores involucrados en la definición, promoción y administración la calidad educativa (Valenzuela, 2009). Este estudio tiene como objetivo determinar y clasificar algunos de los factores que influyen en la calidad de la eduación virtual. Para el logro este fin, se efectuó una revisión sistemática de los factores involucrados en la CEV, se obtuvo una lista de los factores y luego se aplicó la técnica MICMAC para clasificar de forma directa e indirecta en: factores claves, determinantes, autónomos y de resultados. Se utilizó esta técnica por su utilidad para estos casos como se demostró en Martelo, Jimenez-Pitre, \& Villabona-Gómez (2017), donde se determinaron los factores que influyen en la deserción escolar. Igualmente, Martelo, Bastidas, \& Martínez (2018), se utilizó para determinar las variables claves a tener en cuenta para plantear propuestas programáticas en universidades.

La relevancia de esta investigación radica en el aporte de una base de información que permite conocer los factores claves con los cuales se pueden formular estrategias para incrementar la CEV. Este estudio está dirigido a investigadores, docentes, gestores e interesados en la mejora continua de esta modalidad de estudio.

\section{Metodología}

La presente investigación se tipificó como mixta; debido a que se utilizan los enfoques cualitativo y cuantitativo (Hernandéz \& Mendoza, 2018). El diseño fue no experimental, transversal, correlacional y descriptivo. Se consultaron expertos sobre los factores que influyen en CEV, documentos sobre los factores que intervienen en la educación virtual y se realizó un análisis estructural para identificar la naturaleza de la realidad, en el sistema de relaciones entre factores y su estructura dinámica, lo cual es propio del enfoque cualitativo (Herrera, 2017). Para realizar el análisis estructural de estos factores, se implementó la técnica MICMAC (matrices de impactos cruzados Multiplicación Aplicada para una Clasificación), la cual utiliza métodos cualitativos y cuantitativos que permiten identificar los factores o variables claves a través de una matriz de $n \times n$, permitiendo clasificarlas en un plano de cuatro cuadrantes en variables: claves, autónomas, determinantes y de resultados (Arango \& Cuevas , 2014).

La técnica MICMAC permite determinar los factores que inciden en la CEV y la relación de influencia/dependencia que existe entre ellos. Cabe resaltar que la técnica MICMAC estimula la reflexión colectiva, en los resultados no hay una lectura única por lo cual es conviente que el grupo forme su propia interpretación. Por otro lado, los resultados no deben ser tomados como ley, debido al carácter subjetivo del listado y las relaciones entre factores. A continuación, se detallan las fases de la técnica MICMAC. 


\subsection{Fase I. Definición del listado de factores}

En la primera etapa se consideran las variables que determinan el sistema considerado con el fin de obtener un listado homogéneo de variables (internas y externas) al sistema. Se debe realizar una descripción detallada de las variables para favorecer el análisis y la localización en el plano y la descripción de las relaciones entre ellas.

\subsection{Fase II. Descripción de las relaciones entre factores}

Esta fase consiste en el llenado de la matriz, lo cual es de carácter cualitativo. En esta fase interviene el grupo de expertos que previamente hayan estado en el proceso de definición del listado de variables. El procedimiento consiste en plantearse: ¿existe relación de influencia directa entre la variable i y la variable j? Si la respuesta es negativa, la valoración será 0 , en el caso contrario, la pregunta es si esta relación de influencia directa es débil (1), mediana (2), fuerte (3) o potencial (4). Respondiendo estas preguntas se llena la matriz.

\subsection{Fase III. Clasificación de los factores}

En esta fase se identifican las variables clave y se clasifican de manera directa, indirecta y potencial, lo cual permite evidenciar el nivel de relevancia de algunas variables y exponer otras que, por actuar de forma indirecta sobre otras tienen un papel importante que la clasificación directa no permite evidenciar. La clasificación se presenta en un plano de cuatro cuadrantes, donde en el Cuadrante I (Zona de conflicto), se ubican las variables clave; en el Cuadrante II (Zona de poder), se ubican las variables determinantes; en el Cuadrante III (Zona autónoma), se ubican las variables autónomas y en el Cuadrante IV (Zona de salida).

\section{Resultados}

En primer lugar, de la revisión documental se encontraron 17 (diecisiete) factores que, según la literatura consultada, son los más importantes que certifican calidad en la educación virtual y que a continuación se describen: Plataforma educativa, Empatía con el entorno, Atemporalidad didáctica y cumplimiento de tareas, Sólida formación del profesorado, Calidad de los contenidos, Actualidad tecnológica, Cantidad de información, Autogestión de los contenidos de aprendizaje, Frecuencia de las tutorías, Personalización de la educación, Procesos administrativos, Actividades, Metodología de trabajo, Metodologías de evaluación, Flexibilidad del curso, Calidad de Internet y Seguimiento a estudiantes.

Después de la revisión documental, se acudió a la reflexión grupal de los expertos donde se les presentó el listado de 1 os 17 factores para aplicar un segundo filtro en cuanto a los factores de mayor importancia y con ello disminuir el número de factores para optimizar los resultados, debido a que en la técnica MICMAC, entre menor es el número de variables, mayormente se reflejan la relaciones entre ellas. De la reflexión grupal, se obtuvieron 10 (diez) variables, lo cual corresponde a la Fase I de la técnica MICMAC. En la Tabla 1, se puede apreciar el número, el código, el factor y la descripción del factor, En la primera fila, el factor No 1, se identifica con el código ACA, nombre, Autogestión de los contenidos de aprendizaje y como descripción: Condición en la cual el estudiante responde por su aprendizaje, vigila sus objetivos académicos.

Una vez obtenida la lista de los factores, se acude nuevamente a la reflexión colectiva del grupo de experto para que valore y describa las relaciones de motricidad y dependencia de cada factor, lo cual corresponde a la Fase II de la técnica MICACM. A continuación, en la Figura 1, muestra la matriz de influencia/dependencia directa (MID), en la cual se puede observar que, según la reflexión del grupo de expertos, el factor ACC (Autogestión de los contenidos de aprendizaje) mantiene una relación de influencia/dependencia fuerte (3) con el factor ADC (Atemporalidad didáctica y cumplimiento de tareas); una relación de influencia/dependencia fuerte (3) con el factor CC (Calidad de contenidos); una relación de influencia/dependencia nula (0) con el factor $\mathrm{Cl}$ (Calidad del 
internet); una relación de influencia/dependencia fuerte (3) con el factor CE (Empatía con el entorno); y así sucesivamente se describen las relaciones de cada factor.

Tabla 1

Factores que influyen en la CEV

\begin{tabular}{|c|c|c|c|}
\hline No & Código & Variable & Descripción \\
\hline 1 & ACA & $\begin{array}{l}\text { Autogestión de los } \\
\text { contenidos de aprendizaje. }\end{array}$ & $\begin{array}{c}\text { Condición en la cual el estudiante responde por su aprendizaje y } \\
\text { vigila sus objetivos académicos. }\end{array}$ \\
\hline 2 & $A D C$ & $\begin{array}{l}\text { Atemporalidad didáctica y } \\
\text { cumplimiento de tareas. }\end{array}$ & $\begin{array}{l}\text { El tiempo y la presteza con los que el estudiante realiza y cumple } \\
\text { con las tareas. }\end{array}$ \\
\hline 3 & $\mathrm{CC}$ & Calidad de los contenidos. & $\begin{array}{l}\text { Los contenidos deben ser acordes con el curso ofrecido y deben } \\
\text { ser útiles, confiables y oportunos }\end{array}$ \\
\hline 4 & $\mathrm{Cl}$ & Calidad de Internet. & Velocidad del Internet, continuidad del servicio \\
\hline 5 & EE & Empatía con el entorno. & $\begin{array}{l}\text { Capacidad de sentir y racionalizar el trabajo que realizan en el } \\
\text { espacio virtual. }\end{array}$ \\
\hline 6 & FC & Flexibilidad del curso. & Posibilidad de acomodar horarios y priorizar temas \\
\hline 7 & ME & Metodología de trabajo. & $\begin{array}{l}\text { Metodología que utiliza el docente para el proceso de enseñanza- } \\
\text { aprendizaje }\end{array}$ \\
\hline 8 & $\mathrm{PE}$ & Plataforma educativa. & $\begin{array}{c}\text { Herramientas tecnológicas que contribuyen a la educación } \\
\text { reunidas en solo lugar. }\end{array}$ \\
\hline 9 & SE & Seguimiento a estudiantes. & $\begin{array}{c}\text { Acompañamiento que se les da a los estudiantes para verificar el } \\
\text { proceso de enseñanza-aprendizaje }\end{array}$ \\
\hline 10 & SFP & $\begin{array}{l}\text { Sólida formación del } \\
\text { profesorado. }\end{array}$ & $\begin{array}{c}\text { Competencias con las que cuentan los docentes para motivar al } \\
\text { estudiante a permanecer y terminar el ciclo de estudio } \\
\text { entendiendo la complejidad que significa el entorno virtual. }\end{array}$ \\
\hline
\end{tabular}

Fuente: Autores

Figura 1

Matriz de influencia/dependencia directa (MID)

\begin{tabular}{|c|c|c|c|c|c|c|c|c|c|c|c|}
\hline & ACA & $A D C$ & $\mathrm{CC}$ & $\mathrm{Cl}$ & EE & FC & ME & PE & SE & SFP & I.D. \\
\hline ACA & 0 & 3 & 3 & 0 & 3 & 2 & 3 & 0 & 3 & 3 & 20 \\
\hline ADC & 3 & 0 & 0 & 2 & 3 & 3 & 2 & 2 & 3 & 1 & 19 \\
\hline $\mathrm{CC}$ & 3 & 3 & 0 & 2 & 0 & 1 & 3 & 1 & 3 & 2 & 18 \\
\hline $\mathrm{Cl}$ & 3 & 3 & 1 & 0 & 0 & 0 & 0 & 3 & 1 & 2 & 13 \\
\hline EE & 3 & 3 & 1 & 0 & 0 & 2 & 1 & 3 & 3 & 2 & 18 \\
\hline FC & 3 & 3 & 3 & 1 & 0 & 0 & 1 & 0 & 3 & 3 & 17 \\
\hline $\mathrm{ME}$ & 2 & 2 & 3 & 0 & 2 & 3 & 0 & 0 & 3 & 2 & 17 \\
\hline PE & 3 & 3 & 1 & 3 & 3 & 3 & 0 & 0 & 3 & 1 & 20 \\
\hline SE & 3 & 3 & 2 & 0 & 2 & 3 & 2 & 0 & 0 & 2 & 17 \\
\hline SFP & 0 & 2 & 3 & 0 & 3 & 3 & 3 & 2 & 3 & 0 & 19 \\
\hline D.D. & 23 & 25 & 17 & 8 & 16 & 20 & 15 & 11 & 25 & 18 & 178 \\
\hline
\end{tabular}

Fuente: Autores 
Teniendo en cuenta que la Figura 1 representa la matriz de influencia directa, lo siguiente es mostrar la primera clasificación que arroja la técnica MICMAC y que corresponde a las relaciones directas presentadas en el Plano de Influencia Directa (PID) (ver Figura 2). Se puede observar que en el Cuadrante I se ubicaron seis (6) factores, ACA (Autogestión de los contenidos de aprendizaje), ADC (Atemporalidad didáctica y cumplimiento de tareas), SFP (Sólida formación del profesorado), CC (Calidad de los contenidos), FC (Flexibilidad del curso), SE (Seguimiento a estudiantes). En el Cuadrante II (Zona de poder), se ubicaron tres (3) factores, ME (Metodología de trabajo), PE (Plataforma educativa), EE (Empatía con el entorno); en el Cuadrante III se ubicó solo el factor $\mathrm{Cl}$ (Calidad de internet). Finalmente, en el Cuadrante IV no se ubicó ningún factor.

Figura 2

Clasificación de las variables por influencias de dependencias Directas

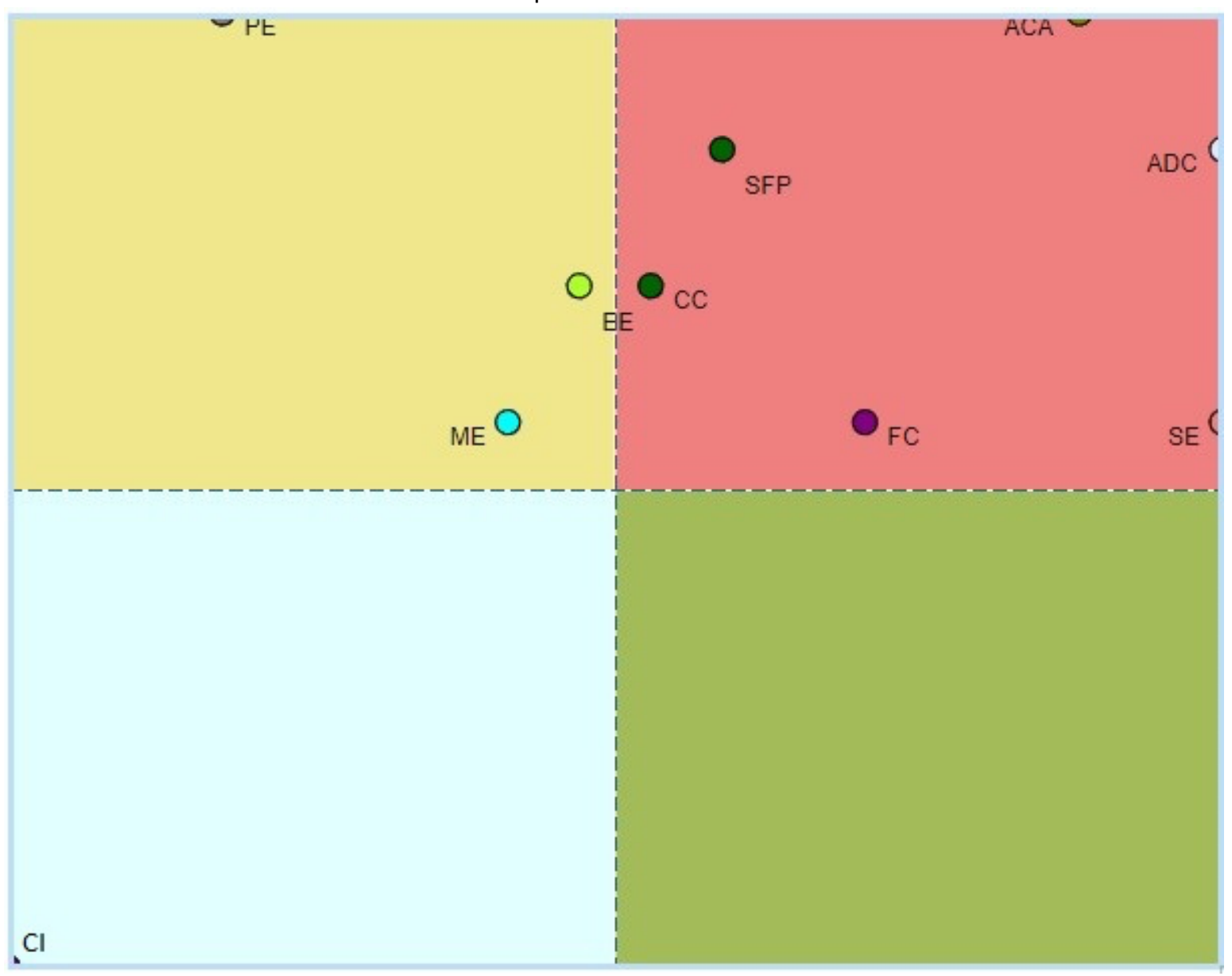

Fuente: Autores

Para la segunda clasificación que se realiza en la Fase III de la técnica MICMAC, se presentan los resultados en la Figura 3, que corresponde al plano de influencia/dependencia indirecta, el cual es el resultado de elevar a la potencia la matriz de influencia/dependencia directa consecuencia de la reflexión colectiva del grupo de expertos. La relevancia de esta clasificación radica en que se evidencia el nivel de importancia de factores que, con la matriz de relaciones directas, no sería posible.

Como se observa en la Figura 3, se reubicaron algunos factores, como es el caso de ME (Metodología de trabajo) y EE (Empatía con el entorno), las cuales, en la clasificación directa, se ubicaron en la Zona de poder, no obstante, la clasificación indirecta las ubicó en la Zona de conflicto. 
Figura 3

Clasificación de las variables por

influencias dependencias Indirectas

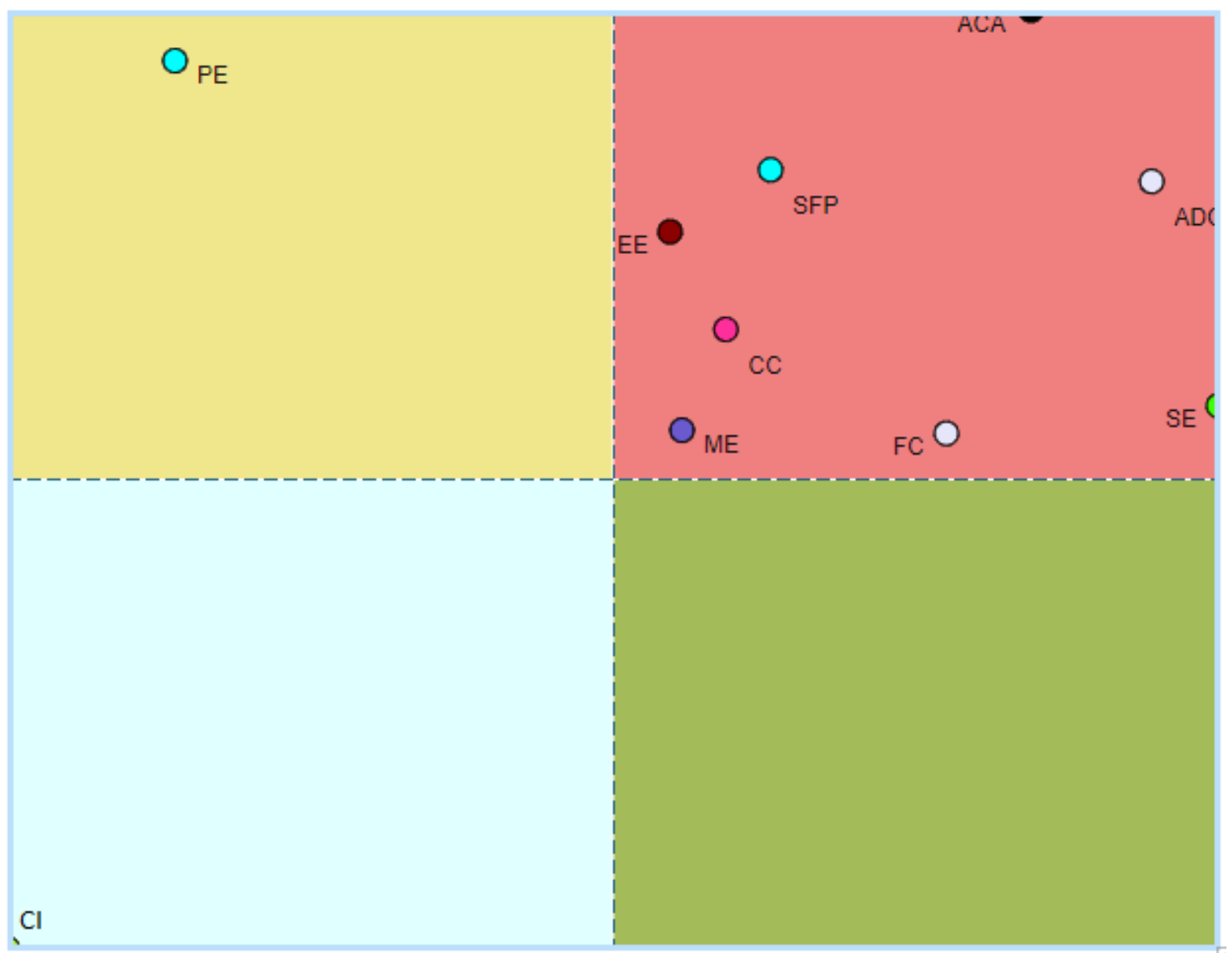

Fuente: Autores

Con base en lo obtenido de la matriz de influencia indirecta, se obtuvo la clasificación indirecta (ver Tabla 2).

Tabla 2

Clasificación de las variables por influencias dependencias indirectas

\begin{tabular}{|l|c|c|}
\hline \multicolumn{1}{|c|}{ Tipo de variable } & Variable & Código \\
\hline Variables clave, estratégicas o de reto & Autogestión de los contenidos de aprendizaje. & ACA \\
\cline { 2 - 3 } & Atemporalidad didáctica y cumplimiento de tareas & ADC \\
\cline { 2 - 3 } & Calidad de contenidos & CC \\
\cline { 2 - 3 } & Metodología de trabajo & ME \\
\cline { 2 - 3 } & Empatía con el entorno & EE \\
\cline { 2 - 3 } & Flexibilidad del curso & FC \\
\cline { 2 - 3 } & Sólida formación del profesorado & SFP \\
\cline { 2 - 3 } & Seguimiento a estudiantes & SE \\
\hline Variables determinantes o "influyentes" & Plataforma Educativa & Cl \\
\hline Variables autónomas o excluidas & Calidad del internet & ---- \\
\hline Variables dependientes o variables de resultado & ---------- & \\
\hline
\end{tabular}

Fuente: Autores 
Considerando lo descrito, la Autogestión de los contenidos de aprendizaje resultó un factor clave, esto es debido a que según Aponte (2011), el desafío del siglo XXI es que las personas aprendan sobre el uso las TIC y puedan autogestionar su conocimiento. Cabe resaltar que la autogestión del conocimiento se desarrolla en un estilo propio e irrepetible, fundamentado en intereses y necesidades de los educandos. Por otro lado, Rodríguez (2009), expone que históricamente el centro de la educación virtual lo ocupan los estudiantes, sujetos activos y responsables, capaces de hacerse cargo autónomamente de su propio aprendizaje. En este sentido, mientras que la responsabilidad, necesidad e interés por parte del alumno no estén presentes, es difícil lograr la CEV.

De igual modo, el factor Atemporalidad didáctica y cumplimiento de tareas se relaciona fuertemente con el anterior, debido a que, dependiendo de las necesidades e interés del estudiante, dedicará tiempo y empeño en realizar de forma enérgica las tareas que se le asignen. Por otro lado, según La Madriz (2016), la Atemporalidad didáctica y cumplimiento de tareas también se relaciona con la deserción del aula virtual, es por lo anterior que este factor resulta clave para la CEV.

Otro de los factores clasificados como clave fue la Calidad de los contenidos, debido a que forma parte de los elementos necesarios para el aprendizaje por su utilidad en procesos de comunicación educativa (Garduño, 2009). Por otro lado, D'antoni (2007) afirma que los contenidos deben ser de alta calidad, para que el alumno desarrolle destrezas que lo conviertan en una persona útil en la sociedad y el grupo profesional al cual esté vinculado. De igual manera, otros autores plantean que es importante confirmar que tales elementos sean oportunos para el alcance del perfil del egresado.

En cuanto al factor Metodología de trabajo que en el plano directo se ubicó entre los determinantes, en el plano indirecto, es el que más cerca está de la diagonal en el plano de la Figura 3, esto indica que este factor es el más importante entre los claves. Tiene sentido que sea de esta manera, debido a que, como lo afirma Varón (2013), la metodología de trabajo del docente debe propender por incentivar a los educandos a que aprendan no sólo los contenidos sino también el aprendizaje mismo, buscando de esta manera, que una de las bases de la educación virtual como lo es el aprendizaje autónomo se mantenga, para poder tener éxito en el contexto de la educación virtual.

Por otro lado, el factor Empatía con el entorno se clasificó de forma directa, como determinante y de forma indirecta resultó uno de los factores clave; tiene sentido que sea así, debido a que es uno de los factores con mayor relevancia en el entorno virtual, como lo demostró el estudio de La Madriz (2016), donde se encontró que una de las decisiones personales por las cuales los estudiantes deciden desertar del aula virtual está relacionada con este factor y con la dificultad para administrar el tiempo de dedicación al aula virtual. En cuanto al factor Flexibilidad del curso resulta ser clave, porque incluye aspectos relacionados con la didáctica. Según Ferreira \& Salamanca (2013), la flexibilidad presupone las estructuras del proceso de enseñanza-aprendizaje y las formas como son seleccionadas, debido a que permite transformar los métodos académicos rígidos, para producir nuevas estrategias, interdependencia de los conocimientos, aumentar el trabajo colaborativo y establecer una nueva organización de los contenidos y de la evaluación.

Otro de los factores claves resultó ser la Sólida preparación del profesorado, lo cual no tiene discusión, debido a que sin una buena preparación del docente, no se puede garantizar el flujo en el proceso de enseñanzaaprendizaje, y por ello disminuye la CEV. Lo anterior lo sustentan Briseño (2014), Cegarra (2013) y De Acosta (2012), al afirmar que la educación virtual se enfrenta a problemas de calidad por la falta de planeación y dominio de indicadores clave, como la planta docente contratada y las estrategias instrumentadas en el proceso de enseñanza-aprendizaje. Por su parte el factor Seguimiento a estudiantes, que también resultó ser clave, es considerado de gran importancia, debido a que permite evaluar si el alumno realmente avanza en su proceso de aprendizaje. Según Gallardo \& Buleje (2011), en la educación virtual, la posibilidad de un seguimiento detallado 
del progreso del alumno, muchas veces proporciona al docente un mayor conocimiento que con los cursos presenciales, permitiendo evidenciar información con la cual se puede mejorar la calidad de la educación.

En cuanto a los factores determinantes, la clasificación indirecta ubicó en la zona de poder al factor Plataforma educativa, el cual toma cada vez más relevancia entre la población estudiantil. Según Martínez-Bahena, LópezEscogido, Escamilla-Regis, \& Alvarez-Monroy (2017), la importancia de las plataformas educativas radica en que los alumnos utilizan estas herramientas como fuente principal para la apropiación de conocimientos significativos, debido a que no se ciñen a un horario determinado para llevar a cabo consultas, acceder a contenidos en cualquier hora y lugar. Las plataformas educativas son también determinantes en el aprendizaje, cuando, por ejemplo, en tiempos en que la pandemia del COVID-19 confinó a todo el mundo en su casa, obligando a todos acostumbrarse a la virtualidad, especialmente en el sector educativo fueron la mejor opción para continuar con el proceso educativo.

Finalmente, el factor Calidad del internet se calificó de forma indirecta, como autónomo, lo cual indica que es un factor independiente del sistema estudiado y que depende de las condiciones socioeconómicas de los alumnos, por tanto, es un factor que no se puede controlar. Según Carabantes , García, \& Beneit (2007), existen diversos mecanismos que garantizan la calidad en Internet, pero tienen algunas carencias y son desconocidos por los usuarios.

Los anteriores fueron los resultados obtenidos de la clasificación indirecta arrojados por la técnica MICMAC, los cuales indican que, para incrementar la calidad de esta modalidad de estudio, se necesita tener presente los factores clasificados como claves, no obstante, también es necesario evaluar otros factores que no se abordan en este estudio, debido a que estos resultados están sujetos a la reflexión colectiva de un grupo de expertos quienes eligieron los factores. Cabe resaltar que los resultados de esta técnica son una orientación de los factores que se deben tener en cuenta para planear estratégicamente mejorar la calidad de la educación y no una regla, esto es debido al carácter cualitativo de la técnica.

\section{Conclusiones}

Considerando lo expuesto, se podrían presentar varias conclusiones. No obstante, lo que más se puede resaltar es que medir la calidad de educación virtual implica grandes retos, debido a la alta cantidad de variables que se deben considerar para su estudio. Con esta investigación se obtuvo una lista de factores que fueron sometidos a una exhaustiva evaluación por parte de expertos, quienes analizaron las relaciones entre ellos para clasificarlos. La clasificación indirecta evidenció que ocho de los diez factores resultaron ser claves, y uno determinante, lo cual indica que en la CEV todas las variables y factores son importantes y se deben tener en cuenta a la hora de formular estrategias para implementar mejoras en esta modalidad de estudio. Cabe resaltar que los resultados de aplicar la técnica MICMAC, son una guía que muestra un acercamiento a la realidad de la situación estudiada, pero no significa que sea una realidad absoluta debido a que la técnica esta sujeta a bases cualitativas como lo es la elección de los factores y la valoración de las relaciones entre estos.

\section{Referencias bibliográficas}

Aponte, H. (2011). Enseñar sin la integración efectiva de las nuevas tecnologías de la información y comunicación es ultimar la autogestión del aprendizaje. VIRTUAL EDUCA. Obtenido de https://scholar. google. es/scholar

Arango, X., \& Cuevas , V. (2014). Método de análisis estructural: matriz de impactos cruzados multiplicación aplicada a una clasificación (MICMAC). México: UANL. Obtenido de http://eprints.uanl.mx/6167/ 
Ardila-Rodríguez, M. (2011). Indicadores de calidad de las plataformas educativas digitales. Educación y Educadores, 14(1), 189-206.

Barberá, E., Badia, A., \& Mominó, J. (2001). Enseñar y aprender a distancia: ¿es posible? Revista de Docencia Universitaria, 1(3). Obtenido de https://revistas.um.es/redu/article/view/11511

Briseño, M. (2014). En la U de G: Propuesta de normatividad para asesores en línea. Revista Mexicana de Bachillerato a Distancia, 2(3), 68-73.

Carabantes , D., García, C., \& Beneit, J. (2007). Evaluación de la calidad en Internet a través del Campus Virtual: el sistema @racne. In III jornada Campus Virtual UCM : Innovación en el Campus Virtual metodologías y herramientas. Complutense, 289-290.

Cegarra, J. (2013). Webquest: estrategia constructivista de Aprendizaje basada en internet. Investigación y postgrado, 23 (1), 73-91.

D'antoni, S. (2007). Recursos educativos abiertos y contenidos para la educación superior abiertos. Revista de Universidad y Sociedad del Conocimiento (una publicación de la Cátedra UNESCO, de e-learning de la UOC), 4(1). Obtenido de http://www.uoc.edu/rusc/4/1/dt/esp/dantoni.html

De Acosta, C. (2012). Educación universitaria en desarrollo y aplicación del talento humano y la gestión del conocimiento. InterSedes, 13(25), 98- 115.

Ferreira, S., \& Salamanca, M. (2013). La flexibilidad didáctica en entornos virtuales de aprendizaje. Virtu@ Imente, 1(2), 45-59.

Gallardo, L., \& Buleje, J. (2011). Importancia de los programas virtuales en la educación superior peruana. Investigación Educativa, 15(27), 13-126.

Garduño, R. (2009). Contenido educativo en el aprendizaje virtual. Investigación bibliotecológica, 23(44), 15-44.

Hernandéz, R., \& Mendoza, C. (2018). Metodología de la investigación: las rutas cuantitativa, cualitativa y mixta. México: McGraw Hill .

Herrera, J. (2017). La investigación cualitativa. México: UDGVirtual. Obtenido de http://biblioteca.udgvirtual.udg.mx/jspui/handle/123456789/1167

La Madriz, J. (2016). Factores que promueven la deserción del aula virtual. Orbis. Revista Científica Ciencias Humanas, 12(35), 18-40.

Martelo, R., Bastidas, M., \& Martínez, J. (2018). Determination of Key Variables for the Program Proposal to Address Aspiring Undergraduate Programs in Public Universities. Contemporary Engineering Science, 11(15), 707 - 717. doi:https://doi.org/10.12988/ces.2018.8253

Martelo, R., Jimenez-Pitre, I., \& Villabona-Gómez, N. (2017). Determinación de factores para deserción de estudiantes en pregrado a través de las técnicas Iluvia de ideas y MICMAC. Espacios, 38(20). doi:ISSN 0798 1015

Martínez-Bahena, E., López-Escogido, D., Escamilla-Regis, D., \& Alvarez-Monroy, L. (2017). La importancia de las plataformas educativas virtuales como herramienta de apoyo a la educación tradicional. Revista de Tecnología y Educación, 1(1), 16-24.

Marúm-Espinosa, E. (2011). Calidad en el servicio en la Educación a Distancia. Una perspectiva desde México. RIED. Revista Iberoamericana de Educación a Distancia, 14(2), 49-62. 
Medina, I. (2014). Estado del arte de las metodologías y modelos de los Objetos Virtuales de Aprendizaje. Entornos, 28, 93-107.

Niño, M. (2018). Educación Virtual. Revista Alethéia IEU. Obtenido de https://revistaaletheia.ieu.edu.mx/documentos/A_opinion/2018/3_Marzo/Art_Op_4.pdf

Rodríguez, R. (2009). Metodología del trabajo académico. Curso SIUP de la especialización en pedagogía para el desarrollo del apren-dizaje autónomo.

Román, M. (2018). Educación virtual en programas de postgrado. Proceedings of the Digital World Learning Conference CIEV 2018 (págs. 14-20). Guatemala: Universidad de San Carlos de Guatemala. Obtenido de http://biblioteca.galileo.edu/tesario/handle/123456789/771

Sánchez, P. (2012). Calidad en elearning. Primer Congreso Virtual Mundial de e-learning (págs. 33-46). Lulu.

Sierra, C. (2011). La educación virtual como favorecedora del aprendizaje autónomo. Panorama, 73-88. Obtenido de https://journal.poligran.edu.co/index.php/panorama/article/view/37/28

Valenzuela, J. (2009). La calidad en la educación virtual:¿ son los estándares una" camisa de fuerza" para las instituciones educativas?. Revista Iberoamericana de Investigación en Educación Superior., 25-39.

Obtenido de https://repositorio.tec.mx/ortec/bitstream/handle/11285/578186/La+calidad+en+la+educacion+virtual.+S on+los+estandares+una+camisa+de+fuerza+para+las+instituciones+educativas. pdf?sequence $=6$

Varón, C. (2013). La educación virtual como favorecedora del aprendizaje autónomo. Revista Panorama, 5(9).

Esta obra está bajo una Licencia Creative Commons Attribución-NoCommercial 4.0 International

(c) BY-NC 